\title{
Predicting Low Toxicity and Scalable Solvent Systems for High Speed Roll-to-Roll Perovskite Manufacturing
}

Richard Swartwout [1], Rahul Patidar [2], Emma Belliveau [1], Benjia Dou [1], David Beynon [2], Peter Greenwood [2], Nicole Moody [3], Dane deQuilettes [1], Moungi Bawendi [3], Trystan Watson [2], Vladimir Bulovic [1]

[1] Research Laboratory of Electronics, Massachusetts Institute of Technology, 77 Massachusetts Avenue, Cambridge, MA 02139, USA.

[2] SPECIFIC, College of Engineering, Swansea University, Bay Campus, SA1 8EN Swansea, UK

[3] Department of Chemistry, Massachusetts Institute of Technology, 77 Massachusetts Avenue, Cambridge, MA 02139, USA.

\section{Broader Impact}

This manuscript introduces solvent toxicity in solar perovskite ink chemistries as a major technoeconomic limitation for the growth of the technology. More specifically, the capital and operational cost of handling such toxic chemicals to maintain a safe working environment can lead to significant added costs. As all record power conversion efficiency devices to date have been solution processed, this represents a major challenge for the perovskite optoelectronic field and of printed electronics as a whole. Knowing this limitation, we propose that solvent selections for ink chemistries should be more quantitative and focus on lowering toxicity. To this end, we show that a Hansen solubility model is effective in predicting ink systems using lower toxicity solvents. We also show that inks formed from this method are applicable for high-speed slot-die coating, limiting the need for long anneal times. These methods and results demonstrate a useful framework for quantitatively engineering solvent systems with reduced toxicity while simultaneously maintaining and surpassing performance. It therefore provides a pathway and major step forward towards the commercialization of solution coated perovskite technologies. 


\section{Abstract}

Printed lead-based perovskite thin films have gained interest due to their potential to be used in high power conversion efficiency photovoltaic (PV) devices, which could be manufactured with scalable roll-to-roll techniques. In industrial scale-up, toxicity of solvents used in perovskite inks can constrain roll-to-roll manufacturing due to the added cost of managing toxic effluents. Due to solvent toxicity few perovskite solution chemistries in published PV structures are scalable to gigawatt production capacity at low cost. In the present study we show that for scalable perovskite thin film production, the use of aprotic polar solvents should be avoided due to their overall toxicity. Compliance with the worldwide worker safety regulations for solvent exposure limits could require additional air handling requirements for some solvents, which in turn would affect the costeffectiveness of PV production. We show that costs associated with handling of hazardous substances can be significant and estimate an added cost of $\phi 3.7 / \mathrm{W}$ for dimethylformamide (DMF) based, commonly used perovskite coating inks. To solve this problem, we quantitatively develop a new perovskite ink solvent system composed entirely of ether and alcohol, which has an effective exposure limit $14 x$ higher than DMF, making it suitable for industrial coating processes. We show that our new ink solvent system is capable of fabricating high efficiency perovskite solar cells processed in one minute on a standard roll-to-roll system. Our work provides a framework for selection of printed perovskite solvents by using solubility models, as well as regulatory and economics arguments.

\section{Introduction}

The performance of photovoltaic (PV) structures made from solution processed lead-halide perovskites recently exceeded $25 \%$ power conversion efficiency ${ }^{1}$, raising the interest in printing and coating deposition techniques for the scale-up production of this solar technology. With scaleup, toxicity of production needs to be examined, however, there is presently little information and 
safety guidelines for perovskite thin film manufacturing. For example, toxicity of lead in perovskite thin films ${ }^{2-6}$ has led to a growing number of publications examining lead-free perovskites ${ }^{5}$, however, there has been limited work on the toxicity of perovskite ink systems on a whole. These ink systems are composed of solvents ${ }^{7-12}$, processing additives ${ }^{13,14}$ and organic and metallic salts ${ }^{15}$ all of which have different effects on the human body ${ }^{3}$. The ink solvents, as the majority component of inks, have the largest potential for health impact during the perovskite film processing, directing us to focus the present study to identifying less toxic solvent compositions. Indeed, toxic chemicals and solvents have been readily used in industrial semiconductor processing with well-known negative health effects ${ }^{16-20}$, so we will use the present study to generalize the perovskite ink solvent selection framework, quantifying the effects of toxicity in perovskite thin-film manufacturing processes. We develop a set of selection rules that leads us to formulate a less toxic, fast crystalizing perovskite ink for use in slot die coating applications.

\section{Toxicity in Manufacturing}

Chemical toxicity can be quantified with several methods, but the most well understood metric is through the lethal dosing of $50 \%$ of a studied population (LD50). A LD50 study is conducted by subjecting test animals (usually rats, rabbits, or guinea pigs) to a chemical of interest ${ }^{21-23}$. An analog for volatile compounds is the lethal concentration to $50 \%$ of the population (LC50). These tests are non-standardized, with dosage timing and methodology being dependent on each specific study. Therefore, LD50 and LC50 are particularly useful for understanding acute toxicity, rather than longer term chronic effects.

In the thin-film coating industry, workers may be exposed to solvents during their working shift over the course of years ${ }^{24}$. For them, LD50 and LC50 are incomplete metrics for understanding if a solvent is safe to use industrially. Instead, in the present study we use government-regulated work exposure limits, which dictate the maximum chemical concentration a worker is allowed to be exposed to before deemed unsafe. Lower exposure limits correlate to a higher toxicity . We 
use these limits in combination with practical constraints present in industrial settings to determine solvent selection rules for coating inks.

\section{Discussion}

In the United States, solvent exposure in industrial settings are regulated federally through the Occupational Safety and Health Administration (OSHA $)^{25}$. OSHA regulates Permissible Exposure Limits (PELs) as well as ventilation requirements in hazardous areas that could expose a worker to toxic or flammable fumes. Regulations are based on academic studies, historical and medical effects on workers ${ }^{24}$, industry voices and recommendations from the National Institute of Occupational Safety and Health, who generate Recommended Exposure Limits (RELs) ${ }^{26}$. There are many other third-party institutions, such as the American Industrial Hygiene Association (AIHA) and the American Conference of Governmental Industrial Hygienists (ACGIH) who recommend chemical limits such as Threshold Limit Values (TLVs) ${ }^{27}$. In Europe, the European Agency for Safety and Health at Work (EU OSHA) also regulates the use of solvents in the European Union as Occupational Exposure Limits (OELs) ${ }^{28}$. Many countries have adopted or have similar exposure limits to the institutions previously mentioned. For the purpose of this discussion, focus will be placed on United States regulatory practices due to regulations being largely federal in nature, ease of access to databases, and exposure limits being similar country to country. A list of United States OSHA standards that apply to the coating industry can be seen

in Table S1. A list of common solvents used in perovskite based thin film research, along with their exposure limits are seen in Table 1. We also include $25 \%$ of the Lower Explosion Limit (LEL) as a useful metric of comparison. This limit represents the controlled maximum concentration in air to avoid a fire. This concentration regulates concentration buildup in recirculating ovens, exhaust piping and other sealed containment. 
Although some common solvents are unregulated from a manufacturing and occupational safety perspective, they may be regulated through an environmental release perspective. In the United States, the Department of Energy (DOE) has created protective action criteria (PACs) to determine exposure severity in the case of chemical release ${ }^{29}$. For the chemicals listed in Table 1, their respective DOE PAC levels are listed in Table $\mathbf{S 2 .}$

Exposure limits place an upper limit on human exposure, but directly translate into an upper manufacturing limit for a given facility size and air handling capacity. Because solvents are continuously evaporating during a coating process, only a limited amount of solvent can be used before factory workers are over exposed due to solvent vapors. In addition, only a limited amount of air can be extracted from a building at any given time before heating, air conditioning (HVAC) and other environmental control costs become too expensive to support. Therefore, a practical upper manufacturing limit exits based on the permissible exposure limit. We calculate this upper limit assuming a 5 micrometer wet film coating thickness, as previously reported ${ }^{30,31}$, and experimental procedures described later. We also assume a single shift 8-hour working day and a 250-day working year. The end product is assumed to be a $15 \%$ peak power conversion efficiency (PCE) PV module. We find that the upper limit to manufacturing productivity (MW/year) for a given volume of production facility $\left(\mathrm{m}^{3}\right)$ is dependent on the air exchange rate of the production facility (1/hour) as well as the exposure limit of the solvent and its liquid molar volume (liters/mol). We plot the material quantities versus the gross building air exchange rate in Figure 1a. Comparing dimethylformamide (DMF) and tetrahydrofuran (THF), two industrial aprotic solvents, with a modest air exchange rate of 4 exchanges per hour, we find that transitioning to a THF based solvent system over a DMF based system can increase the manufacturing capacity of a same sized factory 22 -fold.

This analysis shows that working with more hazardous substances requires additional resources in the form of air handling and building capacity. We sought to determine a minimum added cost 
to a solution printed solar panel given this increase in operating costs (OpEx) and capital costs (CapEx) for air handling. Operating costs were estimated using air flow capacity and cost estimations provided by the United States Department of Energy and Environmental Protection Agency $^{32}$. Capital costs were calculated using a constant 4 air exchanges per hour in a 3-story factory with a 10 -year cost depreciation and a 15\% PCE module. The facility had a nominal cost of $\$ 5000 / \mathrm{m}^{2}$ of floor area. Additional details for the calculation can be found in the supplementary information. These costs, broken down by OpEx, CapEx and total costs are plotted against the PEL can be seen in Figure 1b. For a solvent system that uses DMF as the main component, the overall cost add is $\phi 0.74 / \mathrm{W}(\phi 0.09 / \mathrm{W}$ for OpEx and $\phi 0.65 / \mathrm{W}$ for CapEx). For a solvent system that uses THF, the overall cost add would be $\phi 0.037 / \mathrm{W}(\phi 0.0042 / \mathrm{W}$ for OpEx and $\phi 0.033 / \mathrm{W}$ for CapEx). We note that the operating cost here only includes costs for heating, cooling, and air circulation. It does not include costs for particle filtration, humidity control and general building maintenance. Therefore, the OpEx cost may be artificially low compared to what may be required in a true manufacturing line.

This analysis assumes constant exposure at the PEL over the course of the working shift. Many institutions implement safety factors of $4 x-10 x$ in order to increase worker safety due to unknown and accidental variables. With a modest $5 \mathrm{x}$ safety factor, a DMF solvent system would add $\phi 3.7 \mathrm{~W}$ in HVAC related costs while a THF based solvent would add $\phi 0.18 / \mathrm{W}$. Using the SunShot 2030 sustainable module price of $\$ 0.30 / W^{33}$, HVAC related expenses for a DMF based system would account for $12.3 \%$ of the module cost and a THF based system would account for $0.6 \%$ of the module cost using these safety factors. These costs do not include cost of solvents, cost of materials, interconnection, packaging or tool depreciation and therefore would represent a significant added cost.

Using four air exchanges per hour as a basis and assuming a medium sized factory building of $100,000 \mathrm{~m}^{3}$ ( $\$ 50 \mathrm{M}$ U.S) we show the upper limit to manufacturing in Figure 1c. This cost is 
plotted alongside the slot-die low-flow limit and an estimated extended flow regime ${ }^{34-36}$. DMF systems are highly limited by hazardous vapors despite fundamental coating speeds being much higher. For THF, coating speeds are initially limited by the low-flow limit in the laminar regime rather than hazardous vapor buildup. By using different slot die head geometry, high coating speeds can be realized through the extended coating regime before workers hit the PEL limit.

Since exposure limits are motivated by worker safety, classes of solvents with generally low exposure limits should be considered more hazardous than solvents with high exposure limits. Polar aprotic solvents, small molecule amines as well as chlorinated solvents in general have low PELs and should follow caution. Alcohols and ethers in general have higher PELs. Lastly, the lower explosion limits (LEL) are similar across all solvents, and these values are one to two orders of magnitude above the permissible exposure limit. Therefore, if a solvent vapor is controlled to its permissible exposure limit, it will also be controlled to its fire safety limit (25\% of LEL) given that there is adequate control to prevent solvent buildup in certain high-risk areas, like recirculating ovens.

\section{Engineering Safer Solvent Systems}

As discussed in the previous section, systems with higher permissible exposure limits allow for higher manufacturing limits, increased worker safety and lower costs. We sought to create an ink system with an overall higher exposure limit than traditional DMF systems. For this, we resort to lead-methylamine coordination complex systems that has previously been shown to extend perovskite solubility into acetonitrile ${ }^{37}$ and therefore has the potential to extend solubility into other solvents. We select THF as a solvent of interest because it is a cyclic ether with a high permissible exposure limit, and has the ability to form metal-complexes similar to acetonitrile. With this system we make a few qualitative observations. Firstly, the acetonitrile ink can be diluted with THF with no obvious adverse effects to crystal formation. Secondly, the lead-methylamine complex forms 
in pure THF but the complex itself is only partially soluble, creating a liquid-liquid phase separated system as seen in the supplementary Figure $\mathbf{S 1 .}$

Since the THF-lead amine adduct is expected to be organic-like, we explored the solubility mechanics through the Hansen solubility model. For this, we generate the lead-methylamine adduct in THF at a high concentration and then dilute with a solvent of known Hansen parameters. We then fit this data to a traditional organic Hansen ellipsoid and extract the Hansen parameters and interaction radius. Solubility information and modeled Hansen sphere results can be seen in Figure $\mathbf{2 a}$ and the list of solvents and experimental details seen in the supplementary Information and Table S3. Fitted Hansen sphere parameters can be seen in Table 3. Projections of the polar and hydrogen bonding axes as seen in Figure $\mathbf{2} \mathbf{b}$ and the dispersion and hydrogen bonding axes as seen in Figure 2c show that solubility is largely defined by solvent polarity rather than hydrogen bonding.

We take two points within the Hansen sphere and optimize both formulations for spin coated devices to demonstrate the power of the Hansen Model for predicting usable mixed solvent ink systems. For the first mixed system, we create binary mixtures of Acetonitrile and THF (THF/ACN). Acetonitrile and THF are both aprotic, and methylamine gas dissolved in THF can be sourced easily from common suppliers. Therefore, this solvent system is significantly easier to prepare than previous gas bubbling preparation methods. We then directly replace the Acetonitrile with a binary mixture of THF and Methanol (THF/MeOH). After this replacement, the main solvent system is composed entirely of ether and alcohol. This binary mixture has a higher PEL of 200 ppm vs 40 ppm for pure acetonitrile. Optimized device power conversion statistics from both solvent systems are shown in Figure 3a with stabilized power output of the champion cells in Figure 3b. As shown, the two ink systems show similar performance for a n-i-p style heterojunction PV device. Stabilized power outputs of the champion cells also show similar performance, albeit a slightly longer stabilization time is needed for the THF/MeOH ink system 
which may be attributed to the slightly higher hysteresis. The THF/MeOH optimized ink also utilizes a nucleation modifier to improve morphology. Similar inks that do not use this modifier are shown in Figure $\mathbf{S} 2$ and $\mathbf{S} 3$ but have similar performance characteristics. Due to small differences between these ink formulations, we believe both are optimizable to high performance.

To demonstrate the viability of these inks in a manufacturing setting, we optimized slot die coated devices using the THF/MeOH solvent system. Here we choose a p-i-n structured PV device for ease of fabrication in a roll-to-roll setting. The ink is slot-die coated onto a moving web at 1 meter per minute and dried immediately with an air knife. The dried and crystalized film is passed through a one meter oven with a temperature of $130^{\circ} \mathrm{C}$, allowing for only one minute of annealing. Here we find an average PCE of $10.5 \%$ with a champion device of $13.1 \%$ as shown in Figure 4. Device statistics and additional fabrication methods can be seen in Figure S4. The cells are largely limited by high series resistance originating from higher resistivity $50 \Omega$ /square ITO on PET. Higher efficiency spin-coated devices with lower resistivity ITO show similar scanned performance to the $n-i-p$ structures.

\section{Exposure Limits of Mixed Solvents}

The above results show that it is possible to engineer safer solvent systems by using mixed solvents for Pb-methylamine adduct based solvent systems. The United States OSHA defines that for mixed chemical systems that do not have additive effects, the permissible exposure can be represented by an equivalent exposure as seen in Equation $1^{25}$. Here, $\mathrm{C}$ is the time weighted average exposure concentration of the component and $L$ is the exposure limit. To be compliant the equivalent exposure must be less than one.

$$
\begin{gathered}
E=\frac{C_{1}}{L_{1}}+\frac{C_{2}}{L_{2}}+\cdots \frac{C_{n}}{L_{n}} \\
E \leq 1
\end{gathered}
$$


Using this equivalent exposure, we can calculate an equivalent permissible exposure limit for a given mixed solvent system. For the systems discussed in this work, their equivalent PELs are listed in Table 4, along with more traditional systems found in literature.

In all systems the equivalent PEL is pinned by the lowest regulated component, in this case methylamine. However, the amount is low enough that the equivalent PEL remains high, 14x higher than DMF alone. Future work should focus on the replacement of methylamine with nontoxic ligands to further improve ink safety.

\section{Conclusion}

This work shows the importance of lower toxicity solvents in order to achieve high manufacturing capacity in printed thin film perovskite solar technologies. Hazardous solvent ink systems have lower exposure limits which restricts manufacturing capacity due to the need for additional air handling infrastructure. This additional cost can be significant, adding up to $\phi 3.7 / \mathrm{W}$ on a theoretical module of $15 \%$ power conversion efficiency for a DMF based perovskite layer. Due to this cost we demonstrate that DMF is not compatible with high speed manufacturing and may not be economically sustainable. We engineer a lead-methylamine adduct-based solvent system and apply the Hansen Solubility Model to select new solvent systems with lowered toxicity. We develop two new ink systems: one system that is easier to prepare relative to similar acetonitrile systems, as well as a new ink system composed entirely of THF and methanol. This new ink system is fast drying and easily slot die coated. In addition, this system has a $14 \mathrm{x}$ higher permissible exposure limit than a traditional DMF system. We have also shown high efficiency perovskite PV with both proof-of-concept spin coated as well as slot-die coated device films. Overall, this work aims to provide a quantifiable framework for industry compatible solvent selection for perovskite ink development as needed for scalable perovskite PV manufacturing. 


\section{Conflicts of interest}

There are no conflicts to declare

\section{Acknowledgements}

This project is part funded by the European Union Horizon 2020 research and innovation programme under the Marie Sklodowska-Curie grant agreement No 764787. This work also has recieved funding from the European Regional Development Fund through the Welsh Government and was supported by the Engineering and Physical Sciences Research Council (EPSRC) through the SPECIFIC Innovation and Knowledge Centre (EP/N020863/1). The authors would also like to ackowledge Tata Steel Europe for their support. 
a)

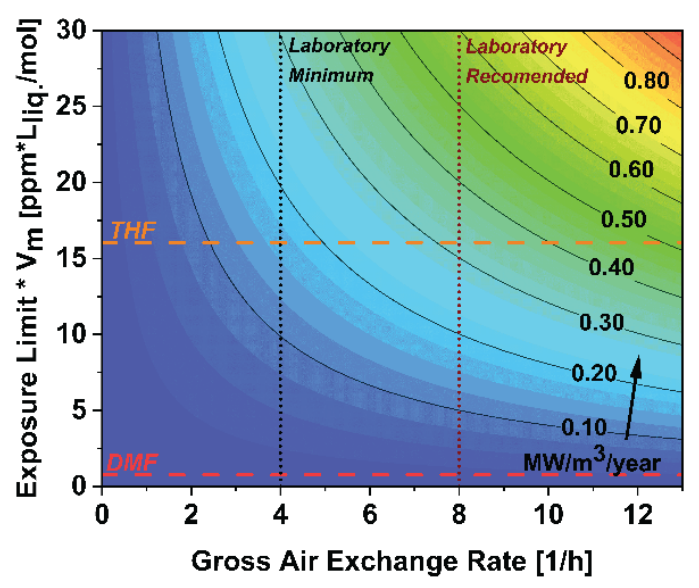

b)

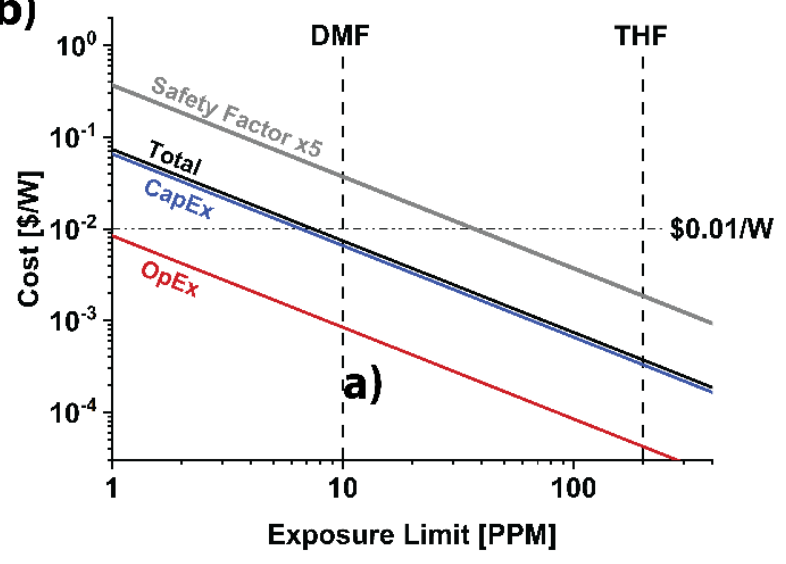

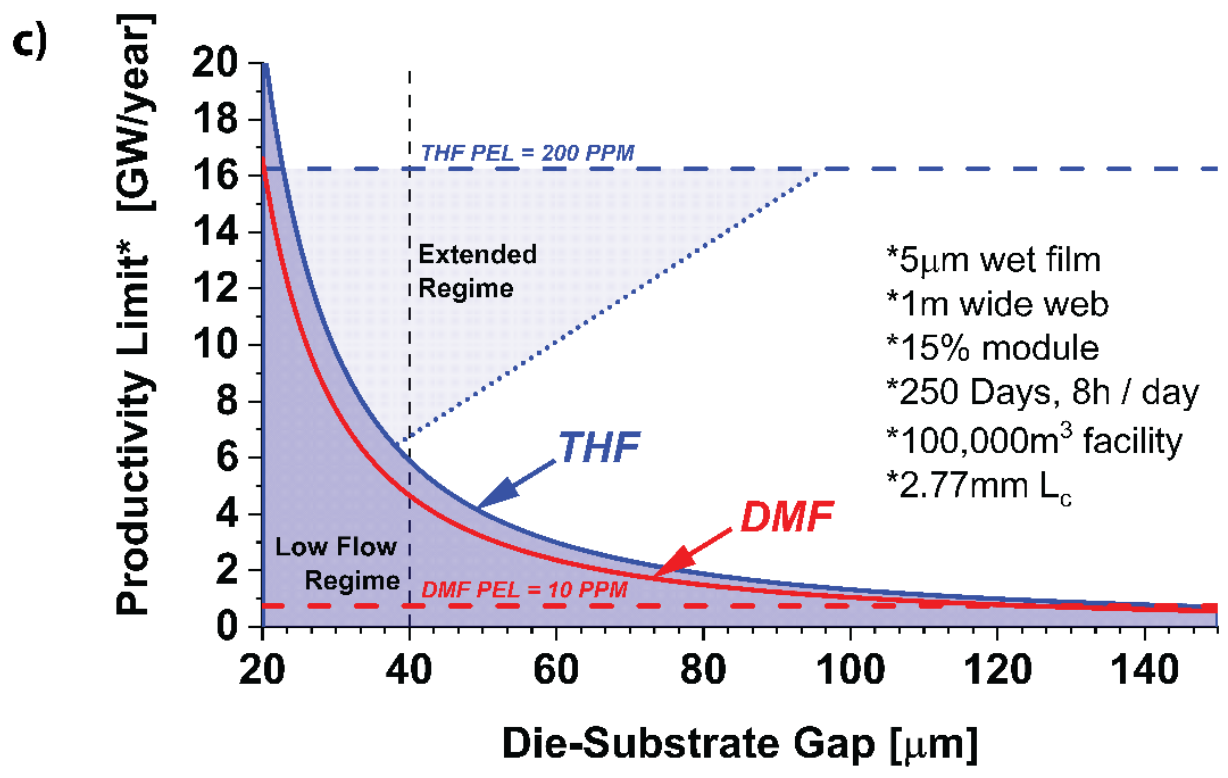

Figure 1: a) Upper manufacturing limit for a given material and the gross air exchange rate. Highlighted are the exposure limits for THF and DMF and OSHA minimum and recommended gross air exchange rates for laboratories. b) Air handling cost for a 15\% PCE printed solar module for a given permissible exposure limit. c) Slot-die microfluidic coating windows compared to productivity exposure limits for THF and DMF solvents. 

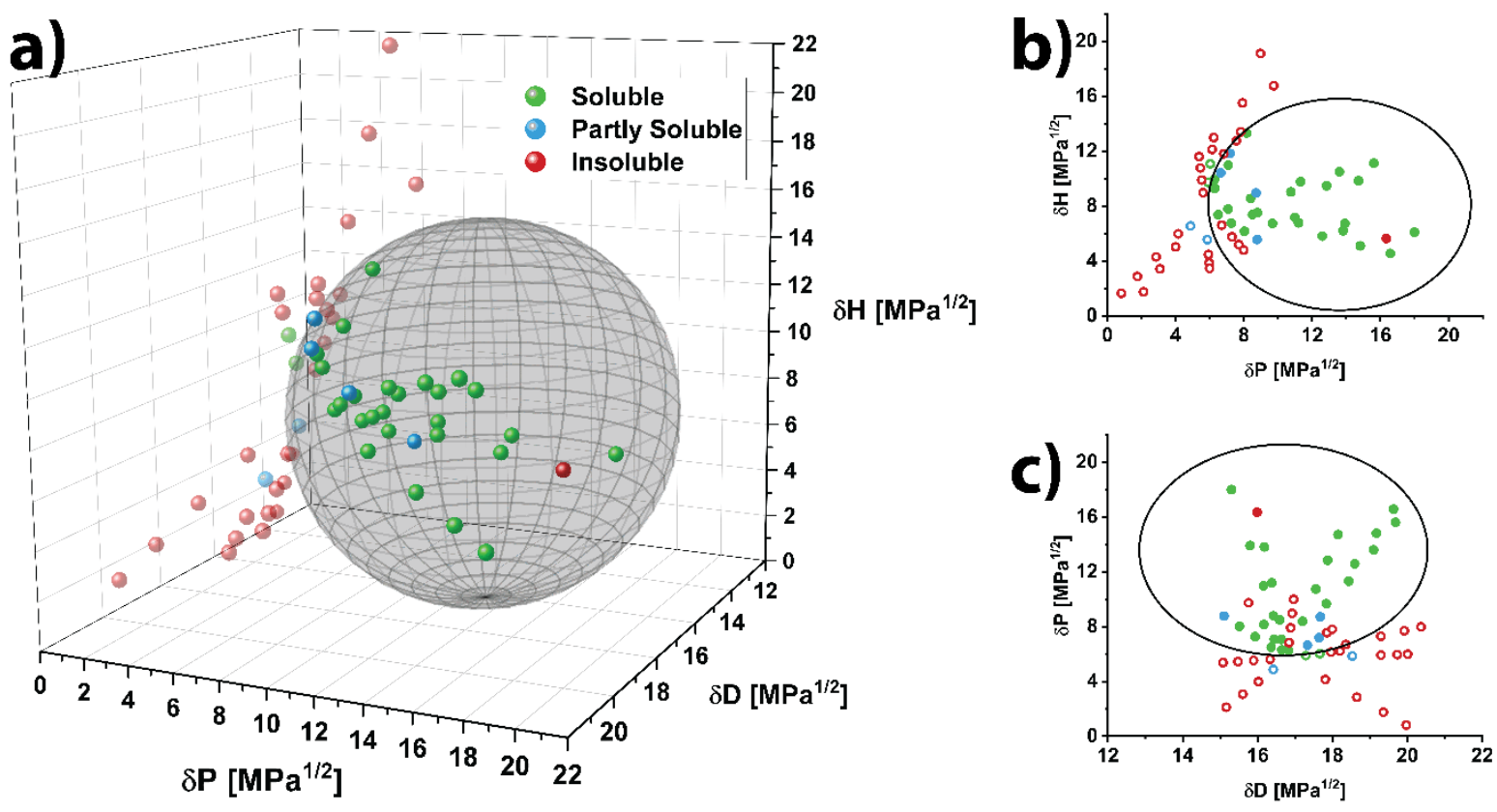

Figure 2: a) 3D Fitted solubility sphere for the THF-MA adduct. b) Projection of the $\boldsymbol{\delta} \boldsymbol{P}$ and $\boldsymbol{\delta} \boldsymbol{H}$ axes. c) Projection of the $\delta \mathrm{D}$ and $\delta \mathrm{P}$ axes. Transparent or hollow points lie outside the solubility sphere. 

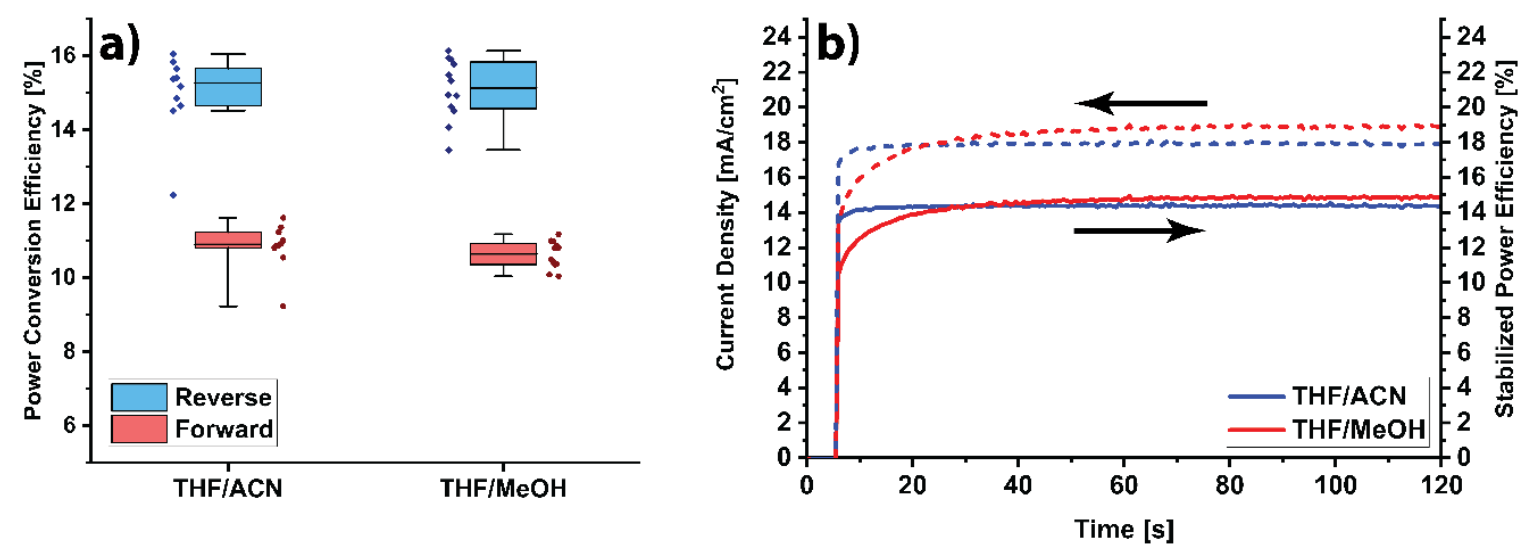

Figure 3: a) Device statistics for THF/ACN and THF/MeOH optimized formulations. b) Stabilized power output and stabilized current density of champion devices.
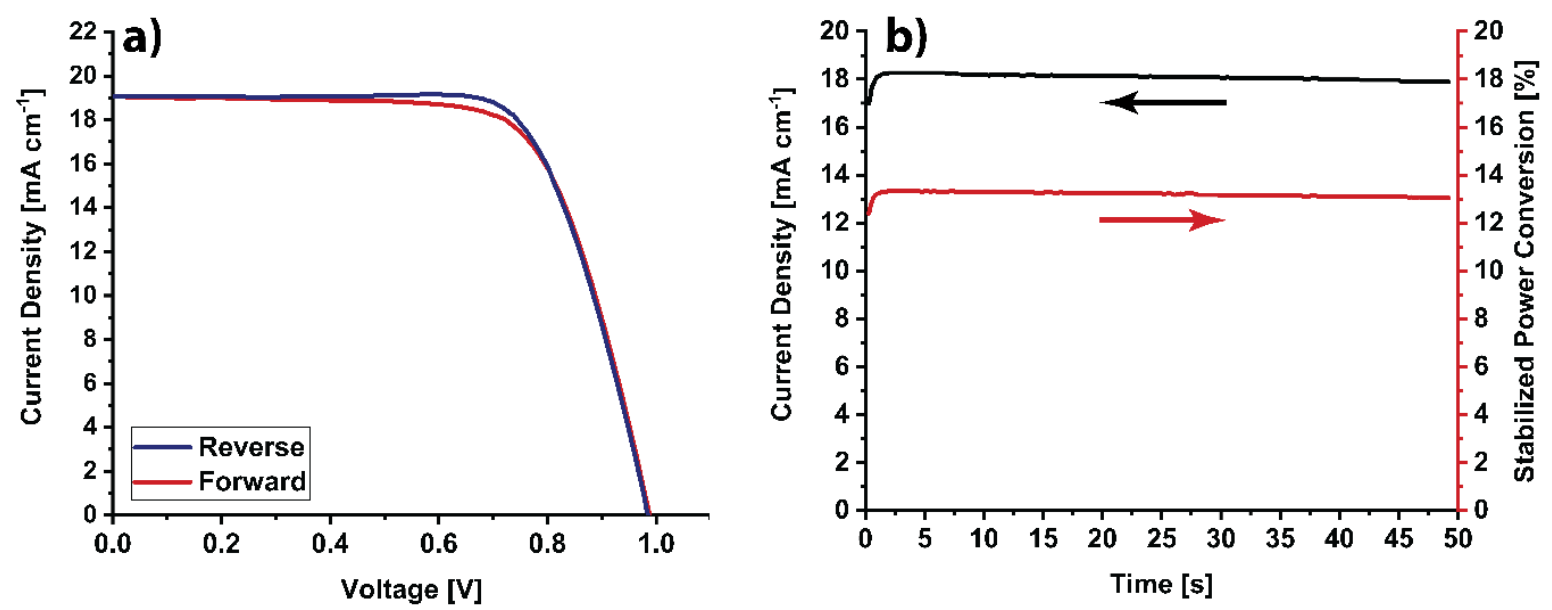

Figure 4: a) J-V curve for champion roll-to-roll coated THF/MeOH device b) Stabilized power output and stabilized current density of champion roll-to-roll device. 
Table 1: Exposure Limits dependent on source. Compiled from issuing institutions ${ }^{26-28}$. Units are PPM (parts-per-million). All values are time weighted over 8 hours except those identified.

\begin{tabular}{|c|c|c|c|c|c|c|}
\hline Solvent & PEL & REL & TLV & OEL & $25 \%$ LEL & Use \\
\hline Dimethylformamide (DMF) & 10 & 10 & 10 & $5 / 10^{[a]}$ & 5500 & Solvent \\
\hline Acetonitrile (ACN) & 40 & 20 & $40 / 60^{[a]}$ & $40 / 70^{[a]}$ & 11000 & Solvent \\
\hline $\begin{array}{r}\text { N-Methyl-2-pyrrolidone } \\
\text { (NMP) }\end{array}$ & --- & --- & --- & $10 / 20^{[a]}$ & 3250 & Solvent \\
\hline $\begin{array}{r}\text { Dimethyl Sulfoxide } \\
\text { (DMSO) }\end{array}$ & --- & --- & --- & --- & 6500 & Solvent \\
\hline gamma-Butyrolactone $^{[\mathrm{c}]}$ & --- & --- & --- & --- & --- & Solvent \\
\hline Propylene Carbonate & --- & --- & --- & --- & 6500 & Solvent \\
\hline 2-Methoxyethanol (2ME) & 25 & 0.1 & 0.1 & 10 & 4500 & Solvent \\
\hline Methylamine ${ }^{[\mathrm{c}]}$ & 10 & 10 & $5 / 15^{[a]}$ & --- & 12250 & Additive \\
\hline Ethylamine & 10 & 10 & $5 / 15^{[a]}$ & 5 & 8750 & Additive \\
\hline Isopropylamine & 5 & --- & $5 / 10^{[a]}$ & --- & 5000 & Additive \\
\hline Pyridine & 5 & 5 & 1 & 5 & 4500 & Additive \\
\hline Chlorobenzene & 75 & --- & 10 & $5 / 15^{[a]}$ & 3250 & Anti-Solvent \\
\hline Chloroform & $50^{[b]}$ & 2 & 10 & 2 & $N / A$ & Anti-Solvent \\
\hline Toluene & $200 / 300^{[b]} / 500^{[a]}$ & 100 & 20 & $50 / 100^{[a]}$ & 2750 & Anti-Solvent \\
\hline Diethyl Ether & 400 & --- & $400 / 500^{[a]}$ & $100 / 200^{[a]}$ & 4750 & Anti-Solvent \\
\hline Tetrahydrofuran (THF) & 200 & $200 / 250^{[a]}$ & $50 / 100^{[a]}$ & $50 / 100^{[a]}$ & 5000 & This Study \\
\hline Methanol & 200 & $200 / 250^{[a]}$ & $200 / 250^{[a]}$ & 200 & 15000 & This Study \\
\hline
\end{tabular}

PEL = Permissible Exposure Limit, REL = Recommended Exposure Limit, TLV = Threshold Limit Value, OEL = Occupational Exposure Limit

LEL = Lower Explosion Limit

[a] Short term exposure limit - 15-30 minutes

[b] Ceiling Limit - exposure may never go over this value

[c] U.S. List 1 precursors. Regulated by the U.S. FDA 

Table 2: Fitted Hansen Parameters for the Pb-Methylamine-THF adduct.

\begin{tabular}{rl}
\hline Parameter & Fit \\
\hline Dispersion - $\boldsymbol{\delta} \boldsymbol{D}$ & $16.69 \pm 0.17$ \\
Polarity - $\boldsymbol{\delta P}$ & $13.60 \pm 0.17$ \\
Hydrogen Bonding - $\boldsymbol{\delta} \boldsymbol{H}$ & $8.12 \pm 0.15$ \\
Interaction Radius - $\boldsymbol{R}_{\mathbf{0}}$ & $7.70 \pm 0.19$ \\
\hline
\end{tabular}

Table 3: Equivalent PEL's using US OSHA's equivalent exposure criteria.

\begin{tabular}{|l|l|l|}
\hline System & PEL-Eq & Ref \\
\hline THF/MeOH/MA & 140 & This work \\
\hline THF/ACN/MA & 50 & This work \\
\hline ACN/MA & 38 & Noel et al. ${ }^{38}$ \\
\hline DMF/DMSO & 17.5 & Saliba et al. ${ }^{39}$ \\
\hline
\end{tabular}




\section{References}

1. Best Research-Cell Efficiency Chart. NREL https://www.nrel.gov/pv/cell-efficiency.html (2020).

2. Moody, N. et al. Assessing the Regulatory Requirements of Lead-Based Perovskite Photovoltaics. Joule 4, 970-974 (2020).

3. Babayigit, A., Ethirajan, A., Muller, M. \& Conings, B. Toxicity of organometal halide perovskite solar cells. Nat. Mater. 15, 247-251 (2016).

4. Binek, A. et al. Recycling Perovskite Solar Cells To Avoid Lead Waste. ACS Appl. Mater. Interfaces 8, 12881-12886 (2016).

5. Ke, W. \& Kanatzidis, M. G. Prospects for low-toxicity lead-free perovskite solar cells. Nat. Commun. 10, 965 (2019).

6. Li, J. et al. Biological impact of lead from halide perovskites reveals the risk of introducing a safe threshold. Nat. Commun. 11, 310 (2020).

7. Gescher, A. Metabolism of N,N-dimethylformamide: Key to the understanding of its toxicity. Chem. Res. Toxicol. 6, 245-251 (1993).

8. Gardner, K. L. et al. Nonhazardous Solvent Systems for Processing Perovskite Photovoltaics. Adv. Energy Mater. 6, 1600386 (2016).

9. Van Hoogen, G. \& Opperhuizen, A. Toxicokinetics of chlorobenzenes in fish. Environ. Toxicol. Chem. 7, 213-219 (1988).

10. Calamari, D., Galassi, S., Setti, F. \& Vighi, M. Toxicity of selected chlorobenzenes to aquatic organisms. Chemosphere 12, 253-262 (1983).

11. Bale, A. S., Barone, S., Scott, C. S. \& Cooper, G. S. A review of potential neurotoxic mechanisms among three chlorinated organic solvents. Toxicol. Appl. Pharmacol. 255, 113-126 (2011).

12. Fowles, J. et al. A review of the toxicological and environmental hazards and risks of tetrahydrofuran. Crit. Rev. Toxicol. 43, 811-828 (2013).

13. Deng, Y. et al. Surfactant-controlled ink drying enables high-speed deposition of perovskite films for efficient photovoltaic modules. Nat. Energy 3, 560-566 (2018).

14. Liu, S. et al. A Review on Additives for Halide Perovskite Solar Cells. Adv. Energy Mater. 10, 1902492 (2020).

15. Mitzi, D. B. Synthesis, Structure, and Properties of Organic-Inorganic Perovskites and Related Materials. in 1-121 (2007). doi:10.1002/9780470166499.ch1.

16. Bauer, S. et al. Toxicological investigations in the semiconductor industry: IV. Studies on the subchronic oral toxicity and genotoxicity of vacuum pump oils contaminated by waste products from aluminum plasma etching processes. Toxicol. Ind. Health 11, 523-41.

17. Schmidt, R. et al. Toxicological investigations in the semiconductor industry: III: Studies on prenatal toxicity caused by waste products from aluminum plasma etching processes. Toxicol. Ind. Health 11, 49-61. 
18. Bauer, S. et al. Toxicological investigations in the semiconductor industry: II. Studies on the subacute inhalation toxicity and genotoxicity of gaseous waste products from the aluminum plasma etching process. Toxicol. Ind. Health 8, 431-44.

19. Bauer, S. et al. Toxicological investigations in the semiconductor industry: I. Studies on the acute oral toxicity of a complex mixture of waste products from the aluminium plasma etching process. Toxicol. Ind. Health 8, 141-56.

20. Kim, M.-H., Kim, H. \& Paek, D. The health impacts of semiconductor production: an epidemiologic review. Int. J. Occup. Environ. Health 20, 95-114.

21. Paget, E. The LD50 test. Acta Pharmacol. Toxicol. (Copenh). 52, 6-19 (2009).

22. Tattersall, M. L. Statistics and the LD50 Study. in 267-270 (1982). doi:10.1007/978-3642-68511-8_46.

23. Parasuraman, S. Toxicological screening. J. Pharmacol. Pharmacother. 2, 74 (2011).

24. de Andrade, V. et al. Occupational risk assessment of paint industry workers. Indian J. Occup. Environ. Med. 15, 52 (2011).

25. Air contaminants - 1910.1000. Occupational Safety and Health Administration https://www.osha.gov/pls/oshaweb/owadisp.show_document?p_id=9991\&p_table=STAN DARDS.

26. OSHA Occupational Chemical Database. Occupational Safety and Health Administration https://www.osha.gov/chemicaldata/ (2020).

27. ACGIH. 2019 TLVs and BEIs. (2019).

28. EU Occupational Exposure Limits (OELs). https://www.chemsafetypro.com/Topics/EU/EU_Occupational_Exposure_Limits_(OELs).h tml (2019).

29. Chemical Saftey Program: PACs for Chemicals of Concern - Index. Department of Energy https://edms.energy.gov/pac/Search (2020).

30. Burkitt, D. et al. Acetonitrile based single step slot-die compatible perovskite ink for flexible photovoltaics. RSC Adv. 9, 37415-37423 (2019).

31. Burkitt, D. et al. Roll-to-roll slot-die coated P-I-N perovskite solar cells using acetonitrile based single step perovskite solvent system. Sustain. Energy Fuels (2020) doi:10.1039/D0SE00460J.

32. Laboratories for the 21st Century: Energy Analysis. (National Renewable Energy Lab. (NREL), 2003).

33. Cole, W. J. et al. SunShot 2030 for Photovoltaics (PV): Envisioning a Low-cost PV Future. http://www.osti.gov/servlets/purl/1392206/ (2017) doi:10.2172/1392206.

34. Larson, R. G. \& Rehg, T. J. Liquid Film Coating. Liquid Film Coating (1997). doi:10.1007/978-94-011-5342-3.

35. Carvalho, M. S. \& Kheshgi, H. S. Low-flow limit in slot coating: Theory and experiments. AIChE J. 46, 1907-1917 (2000).

36. Hens, J. \& Boiy, L. Operation of the bead of a pre-metered coating device. Chem. Eng. 
Sci. 41, 1827-1831 (1986).

37. Noel, N. K. et al. A low viscosity, low boiling point, clean solvent system for the rapid crystallisation of highly specular perovskite films. Energy Environ. Sci. 10, 145-152 (2017).

38. Noel, N. K. et al. A low viscosity, low boiling point, clean solvent system for the rapid crystallisation of highly specular perovskite films. Energy Environ. Sci. 10, 145-152 (2017).

39. Saliba, M. et al. Cesium-containing triple cation perovskite solar cells: improved stability, reproducibility and high efficiency. Energy Environ. Sci. 9, 1989-1997 (2016). 